\title{
Sex Steroids Effects on the Molting Process of the Helminth Human Parasite Trichinella spiralis
}

\author{
Romel Hernández-Bello, ${ }^{1}$ Ricardo Ramirez-Nieto, ${ }^{1}$ Saé Muñiz-Hernández, ${ }^{2}$ \\ Karen Nava-Castro, ${ }^{3,4}$ Lenin Pavón, ${ }^{5}$ Ana Gabriela Sánchez-Acosta, ${ }^{1}$ \\ and Jorge Morales-Montor ${ }^{1}$ \\ ${ }^{1}$ Departamento de Inmunología, Instituto de Investigaciones Biomédicas, Universidad Nacional Autónoma de México, \\ 04510 México, DF, Mexico \\ ${ }^{2}$ Subdirección de Investigación Básica, Instituto Nacional de Cancerología, Secretaría de Salud, 14080 México, DF, Mexico \\ ${ }^{3}$ Departamento de Inmunología e Infectología Pediátrica, Instituto Nacional de Pediatría, Secretaría de Salud, \\ 11000 México, DF, Mexico \\ ${ }^{4}$ Departamento de Biología, Facultad de Química, Universidad Nacional Autónoma de México, 04510, Mexico \\ ${ }^{5}$ Departamento de Psicoinmunología, Instituto Nacional de Psiquiatría "Ramón de la Fuente", 14370 México, DF, Mexico
}

Correspondence should be addressed to Jorge Morales-Montor, jmontor66@biomedicas.unam.mx

Received 28 March 2011; Revised 18 August 2011; Accepted 23 August 2011

Academic Editor: Luis I. Terrazas

Copyright ( 2011 Romel Hernández-Bello et al. This is an open access article distributed under the Creative Commons Attribution License, which permits unrestricted use, distribution, and reproduction in any medium, provided the original work is properly cited.

\begin{abstract}
We evaluated the in vitro effects of estradiol, progesterone, and testosterone on the molting process, which is the initial and crucial step in the development of the muscular larvae (ML or L1) to adult worm. Testosterone had no significative effect on the molting rate of the parasite, however, progesterone decreased the molting rate about a $50 \%$ in a concentration- and time-independent pattern, while estradiol had a slight effect (10\%). The gene expression of caveolin-1, a specific gene used as a marker of parasite development, showed that progesterone and estradiol downregulated its expression, while protein expression was unaffected. By using flow citometry, a possible protein that is recognized by a commercial antiprogesterone receptor antibody was detected. These findings may have strong implications in the host-parasite coevolution, in the sex-associated susceptibility to this infection and could point out to possibilities to use antihormones to inhibit parasite development.
\end{abstract}

\section{Introduction}

The sex steroids hormones, $17 \beta$-estradiol $\left(\mathrm{E}_{2}\right)$, progesterone $\left(\mathrm{P}_{4}\right)$, and testosterone $\left(\mathrm{T}_{4}\right)$, act upon the reproductive system of mammalians by binding to specific sex steroid receptors, which determine changes in reproductive physiology and behaviour $[1,2]$. Recently, it has been shown that sex steroids participate not only in reproductive physiology, but also in a number of different functions, which include immune modulation, brain activity, bone metabolism, and lung and heart physiology. Moreover, also strong direct effects of sex steroids on parasites to modulate different parasite functions have been reported [3-7].

Recent information reveals that sex hormones can affect the course of worm infection [8-12], as in the case of the cestodes Taenia crassiceps and Taenia solium [13-15]. In line with this statement, it is known that the frequency of $T$. solium pig cysticercosis is increased during pregnancy, when there is a significant increase in progesterone levels $[13,16]$. It has also been demonstrated that the castration in naturally infected male boars induces an increase in the prevalence of cysticercosis, which highlights the possible role of host androgens to restrict parasite establishment and estrogens to facilitate it [13]. Furthermore, another helminth, T. crassiceps (a close relative of $T$. solium) has shown to be affected by in vitro sex steroid treatment. Specifically, $17 \beta$-estradiol increases the reproduction of $T$. crassiceps parasites, while testosterone or dihydrotestosterone decreases it [17]. In vivo, when castrated mice are treated with $17 \beta$-estradiol, the number of parasites as well as their infective capacity 


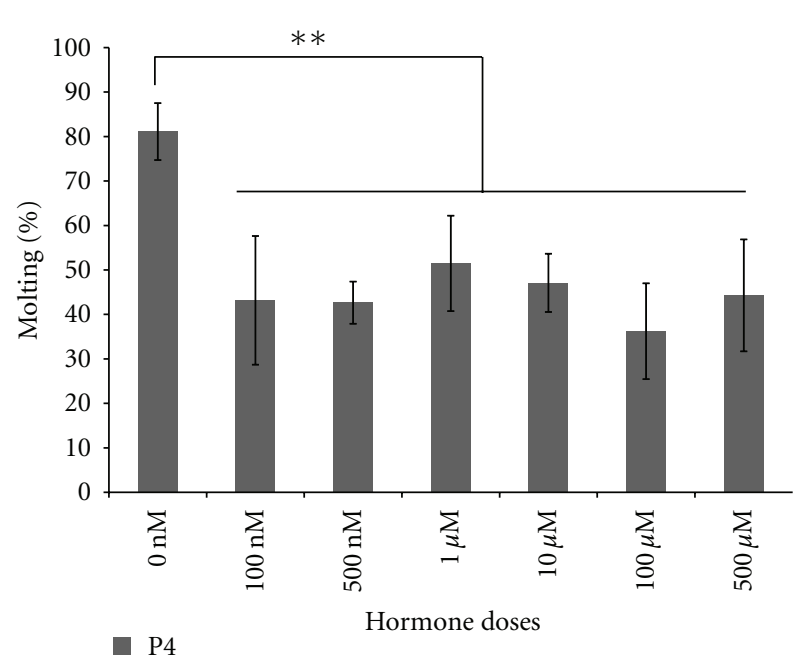

(a)

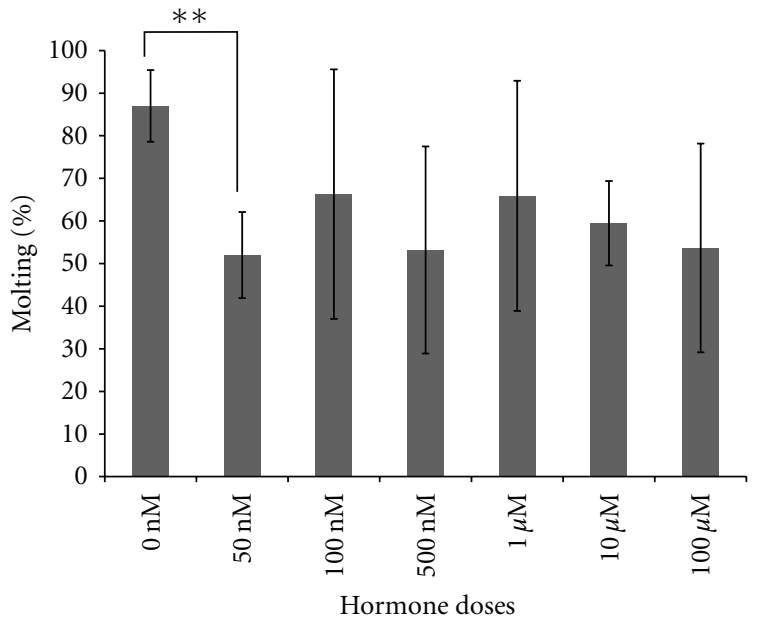

(b)

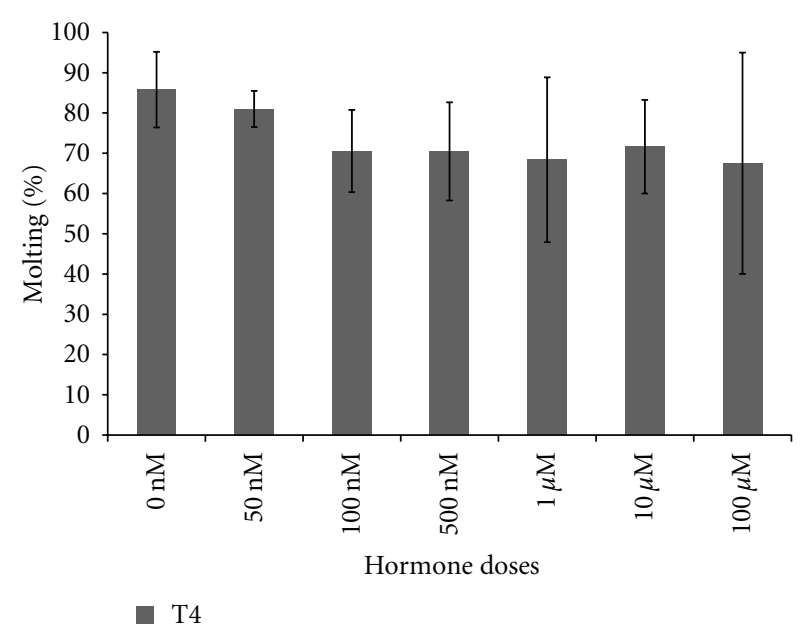

(c)

FIGURE 1: Dose-dependent curves of sex steroid hormones on in vitro molting of muscle larve of T. spiralis. One hundred muscle larve (ML) of T. spiralis were incubated for 36 hours with different concentrations of progesterone (P4), estradiol (E2), or testosterone (T4). Progesterone inhibited the molting rate of the muscle larva of T. spiralis in a concentration-independent pattern (a). Estradiol inhibit only at $50 \mathrm{nM}$ concentration (b), while testosterone has no any effect on molting (c). Each point represents the mean (SD) of quintuplicate determinations of the number of larvae in molting process.

increases up to $200 \%[7,18]$ meanwhile progesterone has the opposite effect in castrated mice of both sexes: a decrease in the parasite loads of almost $100 \%$ [19].

T. spiralis is an intracellular parasitic nematode of mammalian striated muscles. The life cycle of this parasite is completed within a single host and the parasite resides in two distinct intracellular habitats. The infective stage of T. spiralis is part of a nurse cell-larva complex found in striated muscle of prey eaten by carnivores. Digestive enzymes in the stomach release the larva from the muscle tissue and the parasitic L1 migrate to small intestinal sites at the base of villi where they reside in a syncytium of epithelial cells.

Consistent with the level of coevolution evident from parasite adaptation to the host is the assumption that T. spiralis can exploit the hormonal microenvironments within the host [8]. This suggests a system of transregulation (term coined by us) in which the parasite exploits host hormones and growth factors to facilitate infection and potentially increase growth and reproduction rates; this process has been described in at least eight parasitic infections that are caused by both protozoan and metazoans [10]. Furthermore, endocrine factors, related to sex and age, are well recognized as modulators of the immune response $[11,12]$ and by having a direct effect over the parasite. Thus, sex steroid hormones play key roles in the susceptibility to trichinellosis at two levels: (a) protective immune response or (b) direct effect on parasite development $[11,15]$. Steroid hormone effects are not only restricted to cestode parasites, but also to nematodes such as Ancyclostoma dudodenale, whose number of larval and adult stages is increased by sex steroid hormones in several organs of mice [20]. Moreover, adult and muscle larvae of T. spiralis are increased in ovarectomized female 


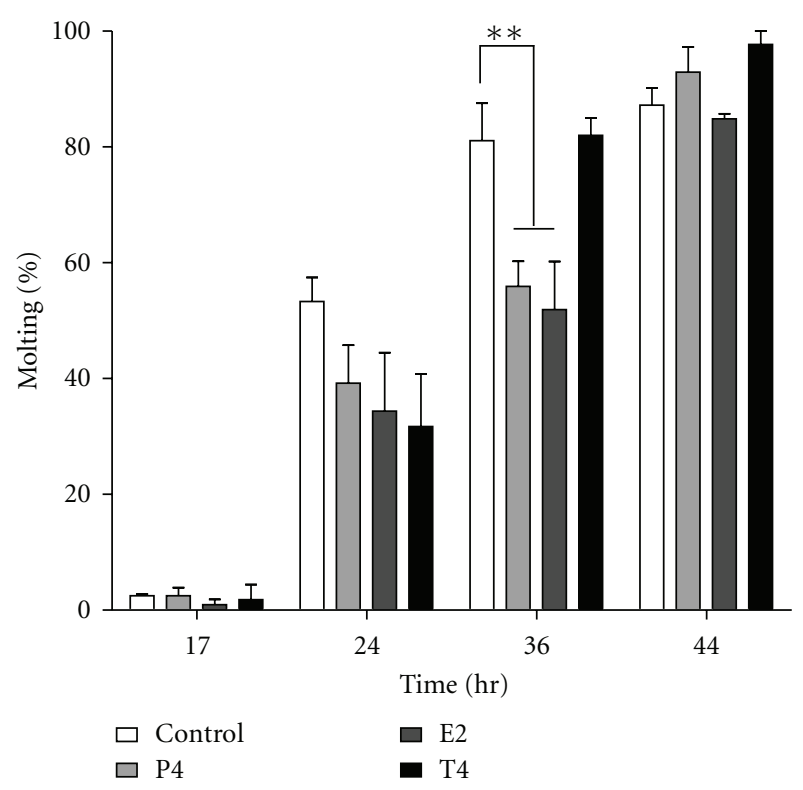

Figure 2: Time curves of T. spiralis molting process after exposure to progesterone (P4), estradiol (E2), and/or testosterone (T4). The parasites were cultured for 44 hours. Progesterone and estradiol had $35-50 \%$ of inhibitory effect on molting rate compared with control and testosterone. The maximum inhibitory effect was at $36 \mathrm{hr}$ of culture. Each point represents the mean (SD) of 5 assays counting the number of molting larvae and the mean was obtained. ${ }^{* *} P<$ 0.05. The hormone concentration was as follows: P4, $100 \mathrm{mM}$; E2, $50 \mathrm{nM}$, and T4, $50 \mathrm{nM}$.

rats [10], suggesting that estrogens and progesterone are restrictive factors for parasite establishment, while androgens should play a permissive role to the infection. In the case of the nematode T. spiralis, males are generally more susceptible than females to the infection, since in mice the males present more parasite burden at both intestinal and muscle level than females do [21-23]. This finding may represent an interesting approach in trichinellosis by $T$. spiralis, because if we know that sex steroids can specifically down-regulate genes involved in the fecundity and oviposition of the parasite, we can propose the use of sex steroid analogous to modulate this effect. Taking into consideration this information, the aim of the present study was to explore the role of sex steroids on T. spiralis development, evaluating its in vitro effects on the molting process, which is the key in the maintenance of the infectious cycle in the host. The in vitro effect of progesterone, estradiol, and testosterone on T. spiralis was studied through pharmacological and molecular (RT-PCR, immunohistochemistry and flow cytometry) approaches, in order to figure out the mechanism of sex steroids actions in the parasite.

\section{Materials and Methods}

2.1. Obtention of Parasites. T. spiralis was maintained in the laboratory by serial passage infections in Wistar rats. The infective-stage ML were recovered as described in [24]. Briefly, the carcass of experimentally infected mice at 30 days of infection was digested by a standard pepsin-hydrochloric acid digestion method to obtain LM stage.

2.2. Sex Steroids Dose-Response Time Curves. Culture grade estradiol, progesterone, and testosterone were obtained from Sigma (Sigma-Aldrich, USA). For in vitro tests, estradiol and progesterone water soluble (Sigma-Aldrich, USA) were dissolved in RPMI 1640 free serum culture medium (Gibco) at desired stock concentration and sterilized by passage through a $0.2 \mathrm{~mm}$ millipore filter. Testosterone was dissolved in absolute ethanol to the desired stock concentration and sterilized by passage through a $0.2 \mathrm{~mm}$ millipore filter. For cultures, the experimental design was as follows: 100 muscular larvae were cultured in 24-well plates and 6 wells per condition were used. Groups designed were as follows: control (only RPMI); control vehicle (EtOH-RPMI); estradiol; progesterone; testosterone. For time-response curves, parasites from all treatments were cultured during $48 \mathrm{hr}$, with medium change at $24 \mathrm{hr}$, using optimal physiological concentration for estradiol $(50 \mathrm{nM})$, progesterone $(100 \mathrm{nM})$, and testosterone $(50 \mathrm{nM})$. Culture wells contained $2 \mathrm{~mL}$ of RPMI medium and were incubated at $37^{\circ} \mathrm{C}$ and $5 \% \mathrm{CO}_{2}$. Steroids were prepared to add $100 \mu \mathrm{L}$ to $5 \mathrm{~mL}$ of the medium in each well to final concentration. From concentrationresponse curves of each steroid, we selected physiological concentration for estradiol, progesterone, and testosterone, as well as, increased concentration of each in pharmacological concentration (above $10 \mu \mathrm{M}$ concentration). Survival and molting were determined under light microscopy using Axiovert Ziess Microscope and 25x Neo Plan objective.

2.3. Morphologic Analysis of T. spiralis Treated with Sex Steroids. T. spiralis ML cultured with or without hormones were observed at different hours under light microscopy using Axiovert Ziess Microscope and 25x Neo Plan objective. The microphotographies generated were modified and contrasted using a software image (Adobe Photoshop CS3, US).

2.4. RNA Extraction of Cultured Parasites in Presence of Sex Steroids. Total RNA was isolated from T. spiralis of each in vitro treatment (positive expression control) using Trizol reagent (Invitrogen, Carlsbad, Calif.). In brief, parasites were homogenized in Trizol reagent $(1 \mathrm{~mL} / 0.1 \mathrm{~g}$ tissue $)$, and $0.2 \mathrm{~mL}$ of chloroform was added per $\mathrm{mL}$ of Trizol. The aqueous phase was recovered after $10 \mathrm{~min}$ of centrifugation at $14,000 \mathrm{~g}$. RNA was precipitated with isopropyl alcohol, washed with 75\% ethanol, and redissolved in RNAsefree water. Total RNA concentration was determined by absorbance at $260 \mathrm{~nm}$ and its purity was verified after electrophoresis on $1.0 \%$ denaturing agarose gel in presence of $2.2 \mathrm{M}$ formaldehyde. Total RNA was stored at $-70^{\circ} \mathrm{C}$

2.5. Immunohistochemistry of Caveolin-1 (CAV-1) in the Parasite. T. spiralis ML obtained at $36 \mathrm{hr}$ of culture with or without the sex steroids were embedded and frozen in tissue freezing medium (Triangle Biomedical Science, USA) at $-70^{\circ} \mathrm{C}$. Four-micrometre-thick sections of parasites were fixed with $4 \%$ paraformaldehyde for $30 \mathrm{~min}$, permeabilised 

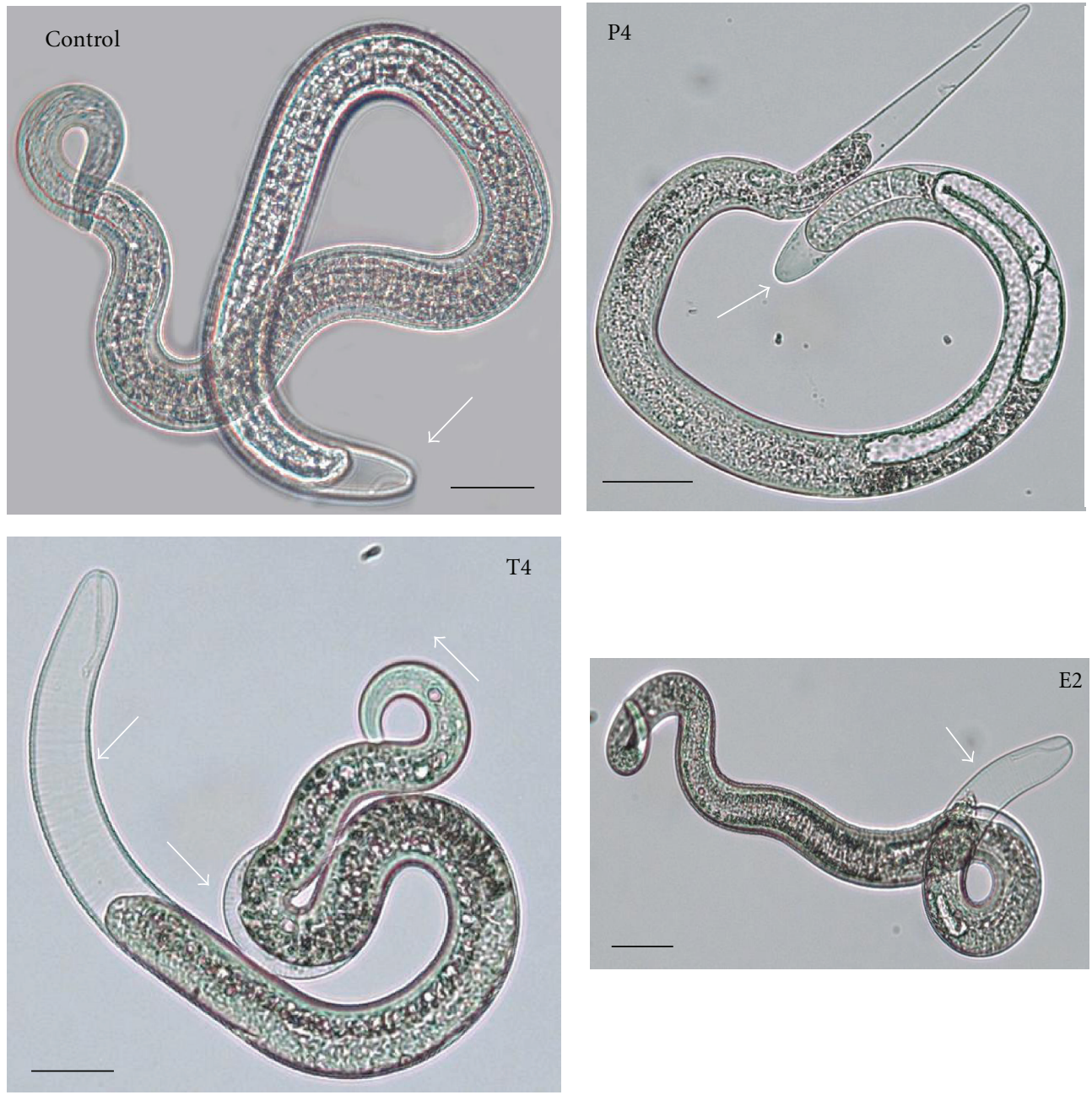

Figure 3: Micrographies of ML parasites cultures in presence of sexual steroids. Parasites cultured at $36 \mathrm{hr}$ with progesterone (P4, $100 \mathrm{mM}$ ), estradiol $(\mathrm{E} 2,50 \mathrm{nM})$, or testosterone $(\mathrm{T} 4,50 \mathrm{nM})$ or in absence of sex steroid (control) were observed in Axiovert microscopy using $25 \mathrm{x}$ objective. In all cases, the old cuticle starts to detach (clear zone) at the apical or basal of the parasite (arrows).

in $1 \%$ PBS-SDS and blocked with RPMI medium containing $0.5 \%$ BSA and 5\% FBS, as described in [25]. Crosssections were incubated with the previously obtained polyclonal anti-Ts-Cav-1 antibody and then incubated with the secondary antibody, fluorescein-isothiocyanate-(FITC-) conjugated goat anti-mouse antibody (Sigma-Aldrich) [25]. Control experiments were performed by incubation secondary antibody. The background fluorescence in samples was reduced by contrast with $0.025 \%$ Evans Blue. Samples were analysed in a Leica TCSSP5 confocal laser-scanning microscope (Leica Microsystems, Germany). The images were constructed using Leica Confocal software.

2.6. Flow Cytometry to Detect Progesterone (PR) Receptor in Parasites Cells. T. spiralis cells were extracted by tissue disruption using a micropestle (Eppendorf, USA) until no more clumps were visible. Cells were centrifuged at $300 \mathrm{~g}$ for $5 \mathrm{~min}$ and recovered in PBS. Cells were stained with the following antibodies for $10 \mathrm{~min}$ at $4^{\circ} \mathrm{C}$ : anti-mouse CD3FITC, and anti-mouse CD19-PE, and anti-mouse Mac-1 (all from BD Biosciences). For intracellular staining, cells were initially fixed with $2 \%$ paraformaldehyde solution for $10 \mathrm{~min}$ at $37^{\circ} \mathrm{C}$, permeabilized with methanol $100 \%$, washed twice with $500 \mu \mathrm{L}$ of staining buffer (PBS pH. 7.4, 2\% Fetal Bovine Serum, $0.02 \% \mathrm{NaN}_{2}$ ), and stained with anticaveolin-1 and anti-PR (Santa Cruz Biotechnology, USA). For primary antibodies recognition, cells were stained with FITC coupled anti-mouse IgG and PE-coupled anti-rabbit IgG for 10 minutes at room temperature, washed, and stored until the analysis is protected from light. Ovary-derived cells were stained with anti-PR as described for T. spiralis cells. All samples were analyzed by flow cytometry using an FACS Calibur (BD, Biosciences, USA) and data analyzed using the FlowJo software.

2.7. Experimental Design and Statistical Analysis. Hormone dose-response time curves were estimated in 4 independent experiments. The response variable used in statistical analyses was the total number of the molted parasites that showed motility in all wells of each hormone concentration 


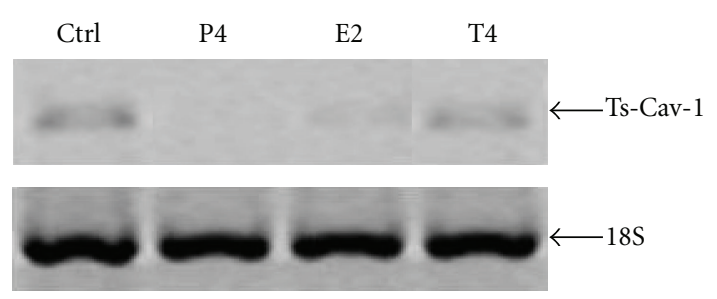

(a)

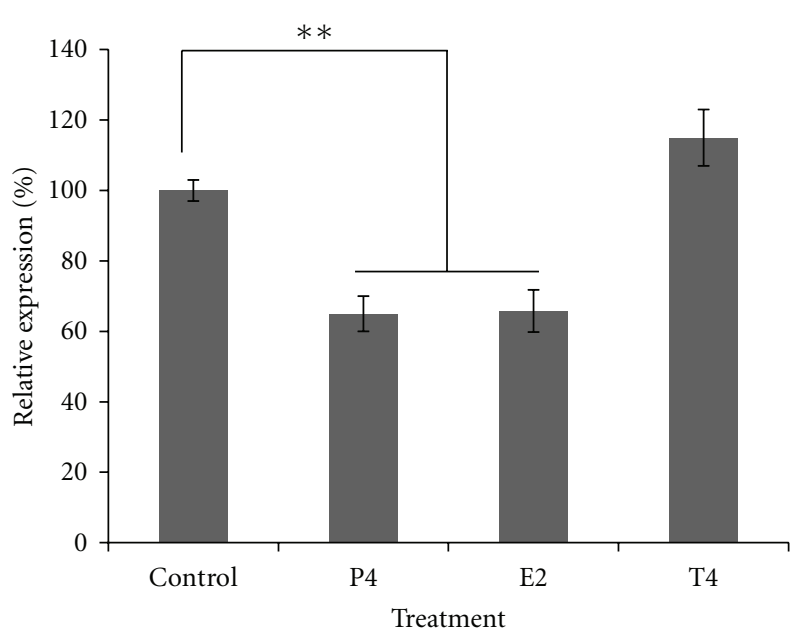

(b)

FIgure 4: Ts-Cav-1 gene expression in T. spiralis ML cultured in presence of sex steroids. A single band of $306 \mathrm{bp}$, corresponding to the caveolin-1 of T. spiralis, was detected in all parasites cultured at $36 \mathrm{hr}$ (a). Progesterone and estradiol reduced the expression level of this gene at least $40 \%$, while testosterone has no effect on the expression of this gene (b). Densitometric analysis is shown (b). $18 \mathrm{~S}$ was used as constitutive expression gene. Data are represented as mean \pm SD. ${ }^{* *} P<0.05$.

and time of exposure, for every experiment. Hormones, their concentrations and times of exposure were the independent variables. The data for the 4 replicates of each treatment were expressed as an average. Data were analyzed using one-way variance analysis (ANOVA). If ANOVA showed significant differences among treatments, a Tukey Test was applied for test significance. Differences were considered significant when $P<0.05$.

2.8. Ethics Statement. Animal care and experimentation practices at the Instituto de Investigaciones Biomédicas are frequently evaluated by the Institute Animal Care and Use Committee, and by governmental (official Mexican regulations (NOM-062-ZOO-1999)), in strict accordance with the recommendations in the Guide for the Care and Use of Laboratory Animals of the National Institutes of Health of the USA, to ensure compliance with established international regulations and guidelines. The protocol was approved by the Committee on the Ethics of Animal Experiments of the Instituto de Investigaciones Biomédicas. Rats were sacrificed to obtain the L1 using sevorane as anesthesia, and all efforts were made to minimize suffering.

\section{Results}

When T. spiralis parasites were in vitro exposed to progesterone, a decrease in the molting process rate about $35-50 \%$ was observed in all treated parasites compared to control groups (Figure 1(a)). However, this molting-inhibiting effect mediated by progesterone was independent of the tested concentrations (Figure 1(a)). For estradiol, an inhibitory effect was observed for the lowest concentration, but just an inhibitory tendency was observed in all cases independent of the culture concentration tested, without any significant differences (Figure 1(b)). In the case of testosterone, there was no apparent effect on the molting of $T$. spiralis in vitro (Figure 1(c)).

Concomitantly, the molting-inhibiting effect of progesterone $(100 \mathrm{nM})$ and estradiol $(50 \mathrm{nM})$ was maintained through all 40 hours of in vitro culture, reaching its highest response on $36 \mathrm{~h}$ in culture, in relation to untreated parasites (Figure 2). Consistently, when parasites were exposed to testosterone ( $50 \mathrm{nM})$, no differences on the percent of molting were observed neither during the first hours of culture nor at the end of the process (Figure 2).

It is important to mention that viability of the molted parasites was verified daily by means of worm motility in the culture plate, which was constant through all days of in vitro culture. Injured parasites were recognized by a progressive internal disorganization: development of clear areas inside of the parasite and increasing number of internal vesicules (Figure 3). Differentiated worms in absence of hormones had a normal development, reaching their tipical shape characteristic of adult at 40 hours in culture and have a normal internal organization and the molting process is ongoing well. Once again, in the presence of estradiol and progesterone, the molting rate is decreased, while in the testosterone-treated parasites, the molt rate is slightly accelerated (Figure 3 ).

In order to look for molecular effects of sex steroids, we sort out the expression of caveolin-1 (Cav-1), which is a marker protein for oocyte development in the female worm of $T$. spiralis. A single band corresponding to the expected molecular weight of the amplified fragment of Cav-1 (approximately $306 \mathrm{bp}$ ) was detected from T. spiralis control and treated with sex steroids (Figure 4). Relative expression of the mRNA of the Cav-1 gene in the parasite revealed that estradiol and progesterone decrease the expression by $40 \%$, while treatment with testosterone increased the same by $10 \%$ (Figure 4). 

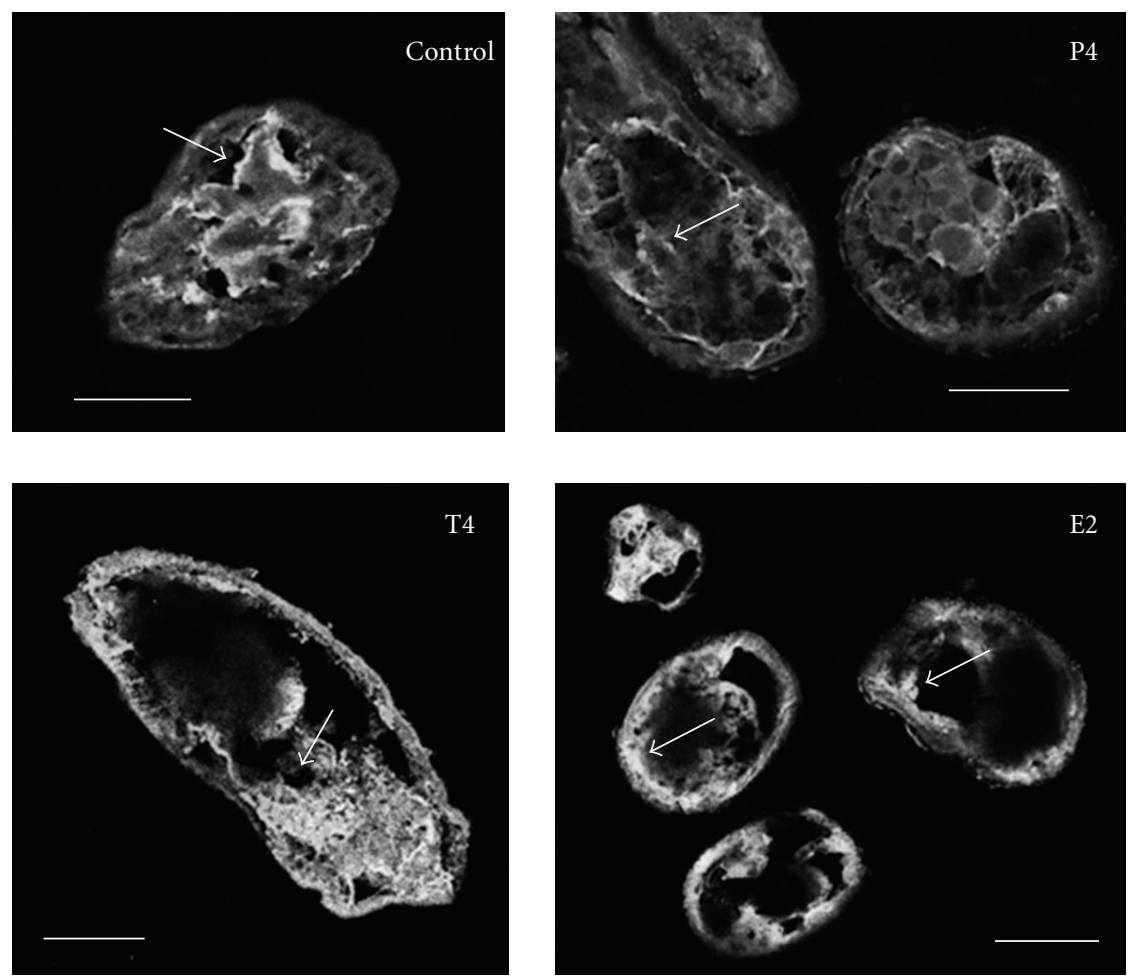

(a)

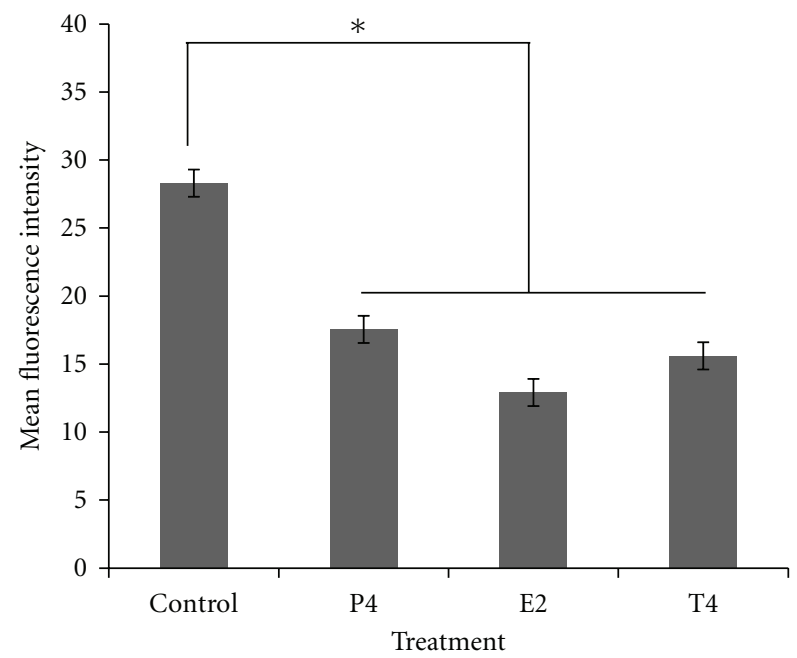

(b)

Figure 5: Indirect immunofluorescence of Ts-Cav-1 protein expression in muscle larvae (ML) cultured. Four-micrometre sections from parasites cultured without or with sex steroids (P4, E2 or T4) were treated with anti-Ts-Cav-1 antibody and fluorescein isothiocyanateconjugated goat anti-mouse antibody and observed under confocal laser microscopy. In all cases of parasite treated there was no changes on Ts-Cav-1 protein expression. Bars $=25 \mu \mathrm{m}$.

Interestingly, when we look for the expression pattern of Cav-1 by immunohistochemistry, there were no apparent differences between the untreated parasites and the ones exposed to sex steroids (Figure 5(a)). When we quantified this pattern of expression, this pattern was confirmed, since quantification of cells expressing Cav-1 was no different when we compared between treatments (Figure 5(b)).
In order to determine if the effect of progesterone was mediated by a putative progesterone receptor in the parasite, we perform flow cytometry using commercial anti-PR antibodies. As shown in Figure 6(a) (upper row), T. spiralis cells do not express immune cell markers such as CD3, CD19, or Mac-1, which are typically present in some types of mammalian leukocytes, which suggest that there 

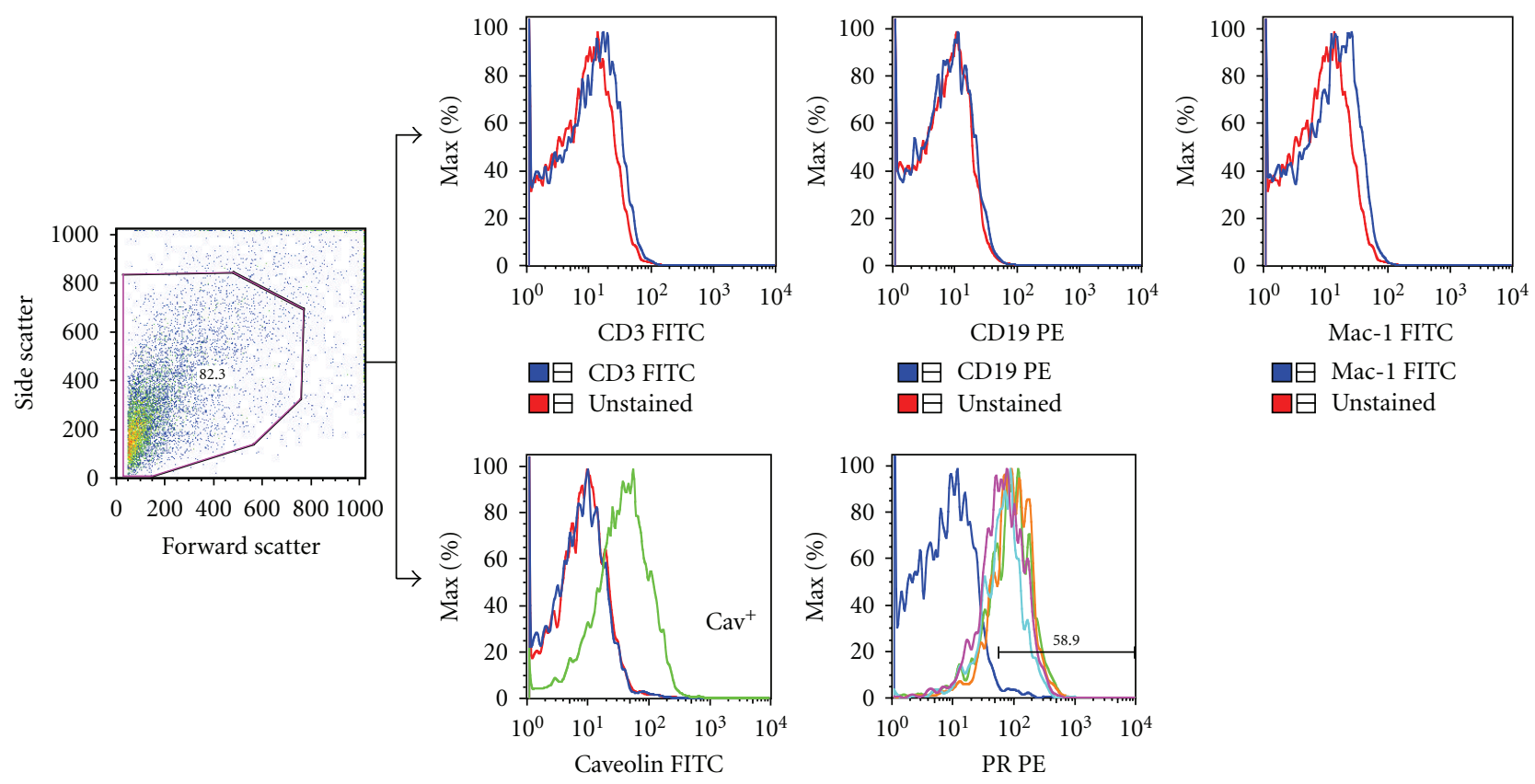

口曰 Mac-1 FITC

$\square$ 曰 Unstained

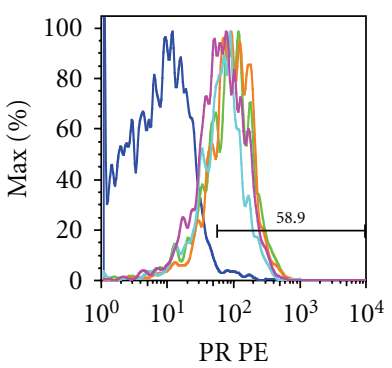

T. spiralis T4 Cav PR

$\square$ 㕷. spiralis P4 Cav PR

$\square \boxminus$ T. spiralis E2 Cav PR

$\square \boxminus$ T. spiralis Media Cav PR

$\square \boxminus$ T. spiralis a-rabbit PE

(a)
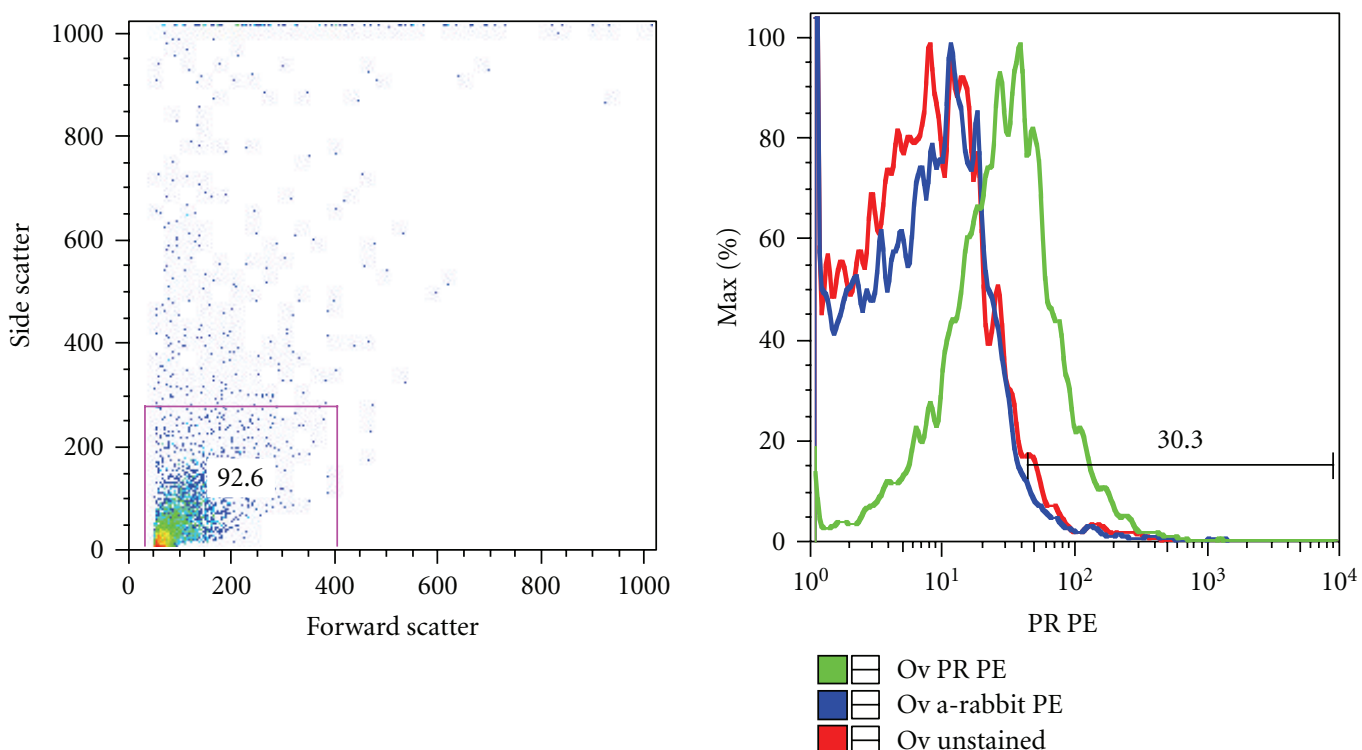

(b)

Figure 6: Progesterone receptor (PR) and host markers expression on T. spiralis cells. (a) T. spiralis cells were stained with anti-mCD3, anti-mCD19, and anti-mMac-1 (upper row); cultured in presence of media (green line), E2 (orange line), P4 (blue line), and T4 (pink line) and stained with anti-Cav-1 of T. spiralis and anti-PR specific antibodies (middle row). (b) Ovary-derived cells were stained with anti-PR antibody as a positive control for PR.

is no contamination with host cells. We then analyzed the expression of the classic progesterone receptor (PR) on parasitic cells that expressed caveolin-1 (a protein cloned, sequenced, and expressed exclusively by this parasite), in response to the steroid hormones estradiol, progesterone, and testosterone. As shown in Figure 6(a) (middle row), $\mathrm{Cav}^{+}$ cells showed a specific binding of anti-PR antibody, which suggested the presence of a PR-like receptor of parasitic 
origin. PR expression was also detected in ovary, as an expression control tissue (Figure 6(b)).

\section{Discussion}

Estrogens and androgens play an important role at different system levels for maintenance of homeostasis in vertebrates. They are implicated not only on the reproductive behavior and physiology, but also act as modulators of immune system, brain activity, and lung and heart physiology. In the last years, increasing information reveals that sex hormones can affect the course of parasite infection $[8,9$, 11,12 ] by modulating the Th1 or Th2 response to lead a susceptibility/resistance to infections. In that way, sex differences on parasitemia can reflect the suppressive effects of testosterone and the increasing effects of estrogens in the immune system [26], principally on Th2 immune responses in females leading to a higher production of interleukins (i.e., IL-4, -5, -6 and -10) [27]. Therefore, males of several species are more susceptible to infections caused by different parasites as well as the prevalence and intensity of parasitic infection than females do $[28,29]$. For example, male mice are less resistant to protozoan like Plasmodium berghei [30] or Trypanosoma cruzi [31] or nematodes like Strongyloides $s p$. [32] than females, while the administration of potent estrogenic compounds as estradiol increased the resistence to the parasite [31-33]. Otherwise, progesterone protects mice from T. crassiceps infections [19].

Particularly on T. spiralis infections, it has been described a resistance/susceptibility associated to gender's host; in which males are more susceptible than females [22, 23]. Interestingly, in pregnant rats, where progesterone levels is increased, the resistance to $T$. spiralis infections throught parasite loads in muscle are increased compared to parasite loads in muscle observed in virgin rats. This effect was mediated by the ability of sera to mediate death in newborn larvae (NBL) of T. spiralis in antibody-dependent cell cytotoxicity assays [34]. Also, progesterone can activate effector peritoneal cells to eliminate NBL in a rapid and antibodyindependent manner [35]. However, the intervention of the host's immune response in dealing with the parasite and the possibility of additional direct effects of sexual steroid hormones on the parasite's physiology should not be hastily discarded. Here we describe the effects of progesterone, estradiol, and testosterone upon the molting process of $T$. spiralis larvae. First of all, it was clear that progesterone has a direct inhibitory effect on the percent of worms that are going through the molting process in vitro. In fact, progesterone exerts a marked molting-inhibitory effect in a concentration-independent pattern, maintained entire time in culture, making that all parasites differentiate at 36 hours of in vitro culture.

T. spiralis parasites not only showed molting, but they also presented a constant motility in the culture plate, which suggests that neither estradiol nor progesterone affected parasite viability and therefore they were alive during the culture process. Moreover, progesterone induced a decrease in the rate of the molting process of the worm in the cultured parasites, with respect to untreated parasites. Estradiol and progesterone inhibited the expression of Ts-Cav-1 in the LM, one gene that is implicated in the maturing and development of the NBL [25]. This observation result is important because it suggests that the LM of $T$. spiralis could modify the expression of specific genes through steroid receptors similar to those found in other invertebrates $[36,37]$. However, no differences on expression of Ts-Cav-1 protein were observed. One explanation is because the Ts-Cav- 1 protein is a molecule that is accumulated in the plasma membrane of the parasite's cells, principally oocytes. We thought that this protein generated in all treatments during the first hours of being cultured, was cumulated in the membrane, and for $36 \mathrm{hr}$ of culture, we do not see any difference in the accumulated protein, although existed a down-regulation in the gene expression at this time on estradiol or progesterone cultures existed. Interestingly, neither percent molting nor molting rate depends on testosterone concentrations. These findings, could explain in part the decreased parasite loads observed in females on infection with T. spiralis and even in pregnant females: sex steroids present in the pregnant female environment avoid the normal rate of molting process on the LM to adult female which directly impacts in the potential adult female that releases NBL. Our results contribute to the knowledge of the mechanisms by which the host microenvironment affects the parasite and parasite protein regulation and expression. As it can be seen, direct effects of sex steroids upon helminth parasites (cestodes, nematodes, and trematodes) are not unusual. In fact, previous results suggest that these pathogens are not only directly affected by hormones, but they have also developed several strategies to exploit the host's endocrine microenvironment $[4,38]$, which include degradation of host proteins as an alternative source of aminoacids [39], development of parasitic-sex steroid receptors $[40,41]$, and cross-activation of signal transduction pathways $[42,43]$.

\section{Conclusions}

Here, we describe different effects of sex steroids that probably have different action mechanisms directly upon T. spiralis. Progesterone effects could explain, at least partially, the low parasite burdens observed on experimental trichinellosis infection in pregnant and in nonpregnant rats [34]. To our knowledge, this is the first report where antitrichinella effects are described for sex steroids and open a promissory field in the design of new strategies that include the antihormone therapy in the control of trichinellosis caused by $T$. spiralis.

\section{Acknowledgments}

Financial support was provided by Grant no. IN 2140113 from the Programa de Apoyo a Proyectos de Innovación Tecnológica, Dirección General de Asuntos del Personal Académico, UNAM. The publication costs of this article were paid by RedFarmed-CONACYT. R. Hernández-Bello has a posdoctoral fellowship from the Instituto de Ciencia y Tecnología del Distrito Federal (ICyT) and from Red 
Farmed-CONACyT. K. Nava-Castro has a posdoctoral fellowship from Dirección General de Asuntos del Personal Académico. The T. spiralis strain was kindly provided by Dr. G. Ortega-Pierres.

\section{References}

[1] D. H. Olster and J. D. Blaustein, "Development of steroidinduced lordosis in female guinea pigs: effects of different estradiol and progesterone treatments, clonidine, and early weaning," Hormones and Behavior, vol. 23, no. 1, pp. 118-129, 1989.

[2] D. W. Pfaff, M. M. Freidin, X. S. Wu-Peng, J. Yin, and Y. S. $\mathrm{Zhu}$, "Competition for DNA steroid response elements as a possible mechanism for neuroendocrine integration," Journal of Steroid Biochemistry and Molecular Biology, vol. 49, no. 4-6, pp. 373-379, 1994.

[3] E. Cabrera-Muñoz, A. González-Arenas, M. Saqui-Salces et al., "Regulation of progesterone receptor isoforms content in human astrocytoma cell lines," Journal of Steroid Biochemistry and Molecular Biology, vol. 113, no. 1-2, pp. 80-84, 2009.

[4] G. Escobedo, C. W. Roberts, J. C. Carrero, and J. MoralesMontor, "Parasite regulation by host hormones: an old mechanism of host exploitation?" Trends in Parasitology, vol. 21, no. 12, pp. 588-593, 2005.

[5] C. Guerra-Araiza, A. Coyoy-Salgado, and I. Camacho-Arroyo, "Sex differences in the regulation of progesterone receptor isoforms expression in the rat brain," Brain Research Bulletin, vol. 59, no. 2, pp. 105-109, 2002.

[6] P. Ascenzi, A. Bocedi, and M. Marino, "Structure-function relationship of estrogen receptor $\alpha$ and $\beta$ : impact on human health," Molecular Aspects of Medicine, vol. 27, no. 4, pp. 299402, 2006 .

[7] L. I. Terrazas, R. Bojalil, T. Govezensky, and C. Larraide, "A role for 17- $\beta$-estradiol in immunoendocrine regulation of murine cysticercosis (Taenia crassiceps)," Journal of Parasitology, vol. 80, no. 4, pp. 563-568, 1994.

[8] G. Escobedo, L. López-Griego, and J. Morales-Montor, "Neuroimmunoendocrine modulation in the host by helminth parasites: a novel form of host-parasite coevolution?" NeuroImmunoModulation, vol. 16, no. 2, pp. 78-87, 2009.

[9] O. Bottasso and J. Morales-Montor, "Neuroimmunomodulation during infectious diseases: mechanisms, causes and consequences for the host," NeuroImmunoModulation, vol. 16, no. 2, pp. 65-67, 2009.

[10] H. S. Thabet, S. S. Thabet, N. M. Ali, and N. S. Ahmed, "Effects of ovariectomy and thyroidectomy on course and outcome of Trichinella spiralis infection in rat," Journal of the Egyptian Society of Parasitology, vol. 38, no. 1, pp. 29-46, 2008.

[11] Y. Osorio, D. L. Bonilla, A. G. Peniche, P. C. Melby, and B. L. Travi, "Pregnancy enhances the innate immune response in experimental cutaneous leishmaniasis through hormonemodulated nitric oxide production," Journal of Leukocyte Biology, vol. 83, no. 6, pp. 1413-1422, 2008.

[12] C. D. Santos, M. P. A. Toldo, F. H. Santello, M. D. V. Filipin, V. Brazão, and J. C. do Prado Júnior, "Dehydroepiandrosterone increases resistance to experimental infection by Trypanosoma cruzi," Veterinary Parasitology, vol. 153, no. 3-4, pp. 238-243, 2008.

[13] J. Morales, T. Velasco, V. Tovar et al., "Castration and pregnancy of rural pigs significantly increase the prevalence of naturally acquired Taenia solium cysticercosis," Veterinary Parasitology, vol. 108, no. 1, pp. 41-48, 2002.
[14] J. Morales-Montor, G. Escobedo, J. A. Vargas-Villavicencio, and C. Larralde, "The neuroimmunoendocrine network in the complex host-parasite relationship during murine cysticercosis," Current Topics in Medicinal Chemistry, vol. 8, no. 5, pp. 400-407, 2008.

[15] N. Peña, J. Morales, J. Morales-Montor et al., "Impact of naturally acquired Taenia solium cysticercosis on the hormonal levels of free ranging boars," Veterinary Parasitology, vol. 149, no. 1-2, pp. 134-137, 2007.

[16] F. W. Bazer, R. C. Burghardt, G. A. Johnson, T. E. Spencer, and $\mathrm{G}$. Wu, "Interferons and progesterone for establishment and maintenance of pregnancy: interactions among novel cell signaling pathways," Reproductive Biology, vol. 8, no. 3, pp. 179-211, 2008.

[17] G. Escobedo, C. Larralde, A. Chavarria, M. A. Cerbón, and J. Morales-Montor, "Molecular mechanisms involved in the differential effects of sex steroids on the reproduction and infectivity of Taenia crassiceps," Journal of Parasitology, vol. 90, no. 6, pp. 1235-1244, 2004.

[18] J. Morales-Montor, S. Baig, C. Hallal-Calleros, and R. T. Damian, "Taenia crassiceps: androgen reconstitution of the host leads to protection during cysticercosis," Experimental Parasitology, vol. 100, no. 4, pp. 209-216, 2002.

[19] J. A. Vargas-Villavicencio, C. Larralde, and J. Morales-Montor, "Gonadectomy and progesterone treatment induce protection in murine cysticercosis," Parasite Immunology, vol. 28, no. 12, pp. 667-674, 2006.

[20] I. Bhai and A. K. Pandey, "Gonadal hormones in experimental Ancylostoma caninum infections in male Swiss albino mice," International Journal for Parasitology, vol. 12, no. 6, pp. 589591, 1982.

[21] L. Charniga, G. L. Stewart, G. W. Kramar, and J. A. Stanfield, "The effects of host sex on enteric response to infection with Trichinella spiralis," Journal of Parasitology, vol. 67, no. 6, pp. 917-922, 1981.

[22] J. J. Reddington, G. L. Stewart, G. W. Kramar, and M. A. Kramar, "The effects of host sex and hormones on Trichinella spiralis in the mouse," Journal of Parasitology, vol. 67, no. 4, pp. 548-555, 1981.

[23] V. Figallová and J. Prokopic, “The effects of host sex and sex hormones on Trichinella spiralis Owen, 1835 and T. pseudospiralis Garkavi, 1972 in the mouse," Folia Parasitologica, vol. 35, no. 1, pp. 59-66, 1988.

[24] D. T. Dennis, D. D. Despommier, and N. Davis, "Infectivity of the newborn larva of Trichinella spiralis in the rat," Journal of Parasitology, vol. 56, no. 5, pp. 974-977, 1970.

[25] R. Hernández-Bello, R. M. Bermúdez-Cruz, R. FonsecaLiñán et al., "Identification, molecular characterisation and differential expression of caveolin-1 in Trichinella spiralis maturing oocytes and embryos," International Journal for Parasitology, vol. 38, no. 2, pp. 191-202, 2008.

[26] M. K. Angele, M. G. Schwacha, A. Ayala, and I. H. Chaudry, "Effect of gender and sex hormones on immune responses following shock," Shock, vol. 14, no. 2, pp. 81-90, 2000.

[27] C. W. Roberts, W. Walker, and J. Alexander, "Sex-associated hormones and immunity to protozoan parasites," Clinical Microbiology Reviews, vol. 14, no. 3, pp. 476-488, 2001.

[28] S. L. Klein, "The effects of hormones on sex differences in infection: from genes to behavior," Neuroscience and Biobehavioral Reviews, vol. 24, no. 6, pp. 627-638, 2000.

[29] M. Zuk and K. A. McKean, "Sex differences in parasite infections: patterns and processes," International Journal for Parasitology, vol. 26, no. 10, pp. 1009-1024, 1996. 
[30] A. B. Kamis and J. B. Ibrahim, "Effects of testosterone on blood leukocytes in Plasmodium berghei-infected mice," Parasitology Research, vol. 75, no. 8, pp. 611-613, 1989.

[31] E. M. de Souza, M. Rivera, T. C. Araújo-Jorge, and S. L. de Castro, "Modulation induced by estradiol in the acute phase of Trypanosoma cruzi infection in mice," Parasitology Research, vol. 87, no. 7, pp. 513-520, 2001.

[32] J. C. Rivero, Y. Inoue, N. Murakami, and Y. Horii, "Androgenand estrogen-dependent sex differences in host resistance to Strongyloides venezuelensis infection in Wistar rats," Journal of Veterinary Medical Science, vol. 64, no. 6, pp. 457-461, 2002.

[33] R. M. F. Libonati, M. G. Cunha, J. M. Souza et al., "Estradiol, but not dehydroepiandrosterone, decreases parasitemia and increases the incidence of cerebral malaria and the mortality in Plasmodium berghei ANKA-infected CBA mice," NeuroImmunoModulation, vol. 13, no. 1, pp. 28-35, 2006.

[34] G. Nuñez, T. Gentile, M. Calcagno, and S. Venturiello, "Increased parasiticide activity against Trichinella spiralis newborn larvae during pregnancy," Parasitology Research, vol. 88, no. 7, pp. 661-667, 2002.

[35] G. G. Nuñez, T. Gentile, S. N. Costantino, M. I. Sarchi, and S. M. Venturiello, "In vitro and in vivo effects of progesterone on Trichinella spiralis newborn larvae," Parasitology, vol. 131, no. 2, pp. 255-259, 2005.

[36] A. Barrabes, J. Goma-Mouanda, F. Reynouard, and C. Combescot, "17 beta-estradiol receptors in Schistosoma mansoni. Contribution to the explanation of the protective power of this hormone in Schistosoma mansoni bilharziasis in the mouse. Preliminary study," Annales de Parasitologie Humaine et Comparee, vol. 61, no. 6, pp. 637-641, 1986.

[37] T. E. Hood, E. J. Calabrese, and B. M. Zuckerman, "Detection of an estrogen receptor in two nematode species and inhibition of binding and development by environmental chemicals," Ecotoxicology and Environmental Safety, vol. 47, no. 1 , pp. 74-81, 2000.

[38] R. T. Damian, "Parasite immune evasion and exploitation: reflections and projections," Parasitology, vol. 115, pp. S169S175, 1997.

[39] M. Shibayama, J. D. J. Serrano-Luna, S. Rojas-Hernández, R. Campos-Rodríguez, and V. Tsutsumi, "Interaction of secretory immunoglobulin A antibodies with Naegleria fowleri trophozoites and collagen type I," Canadian Journal of Microbiology, vol. 49, no. 3, pp. 164-170, 2003.

[40] F. Remoué, J. C. Mani, M. Pugnière, A. M. Schacht, A. Capron, and G. Riveau, "Functional specific binding of testosterone to Schistosoma haematobium 28-kilodalton glutathione Stransferase," Infection and Immunity, vol. 70, no. 2, pp. 601605, 2002.

[41] C. Konrad, A. Kroner, M. Spiliotis, R. Zavala-Góngora, and K. Brehm, "Identification and molecular characterisation of a gene encoding a member of the insulin receptor family in Echinococcus multilocularis," International Journal for Parasitology, vol. 33, no. 3, pp. 301-312, 2003.

[42] M. Spiliotis, C. Konrad, V. Gelmedin et al., "Characterisation of EmMPK1, an ERK-like MAP kinase from Echinococcus multilocularis which is activated in response to human epidermal growth factor," International Journal for Parasitology, vol. 36, no. 10-11, pp. 1097-1112, 2006.

[43] K. Brehm and M. Spiliotis, "The influence of host hormones and cytokines on echinococcus multilocuiaris signalling and development," Parasite, vol. 15, no. 3, pp. 286-290, 2008. 

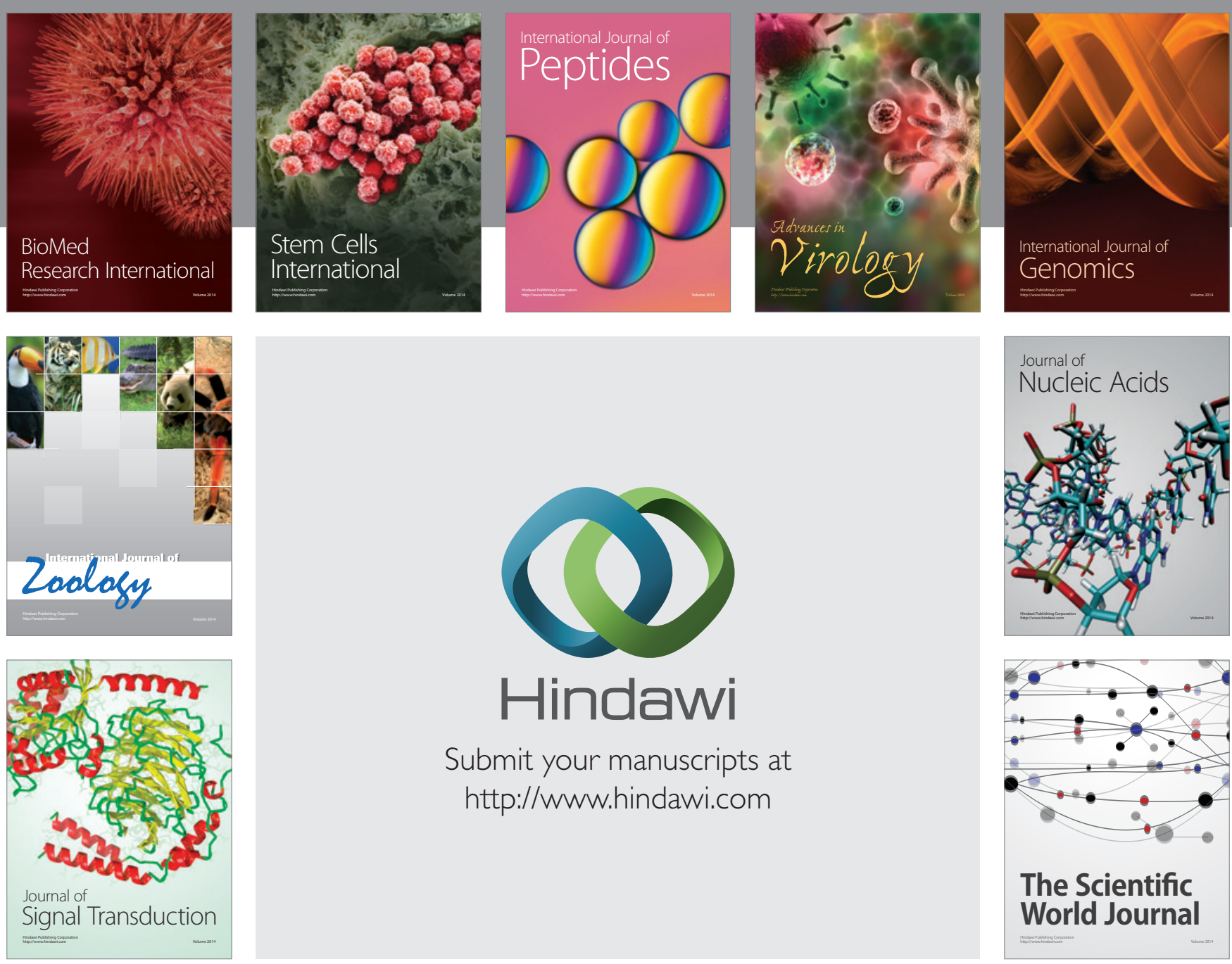

Submit your manuscripts at

http://www.hindawi.com
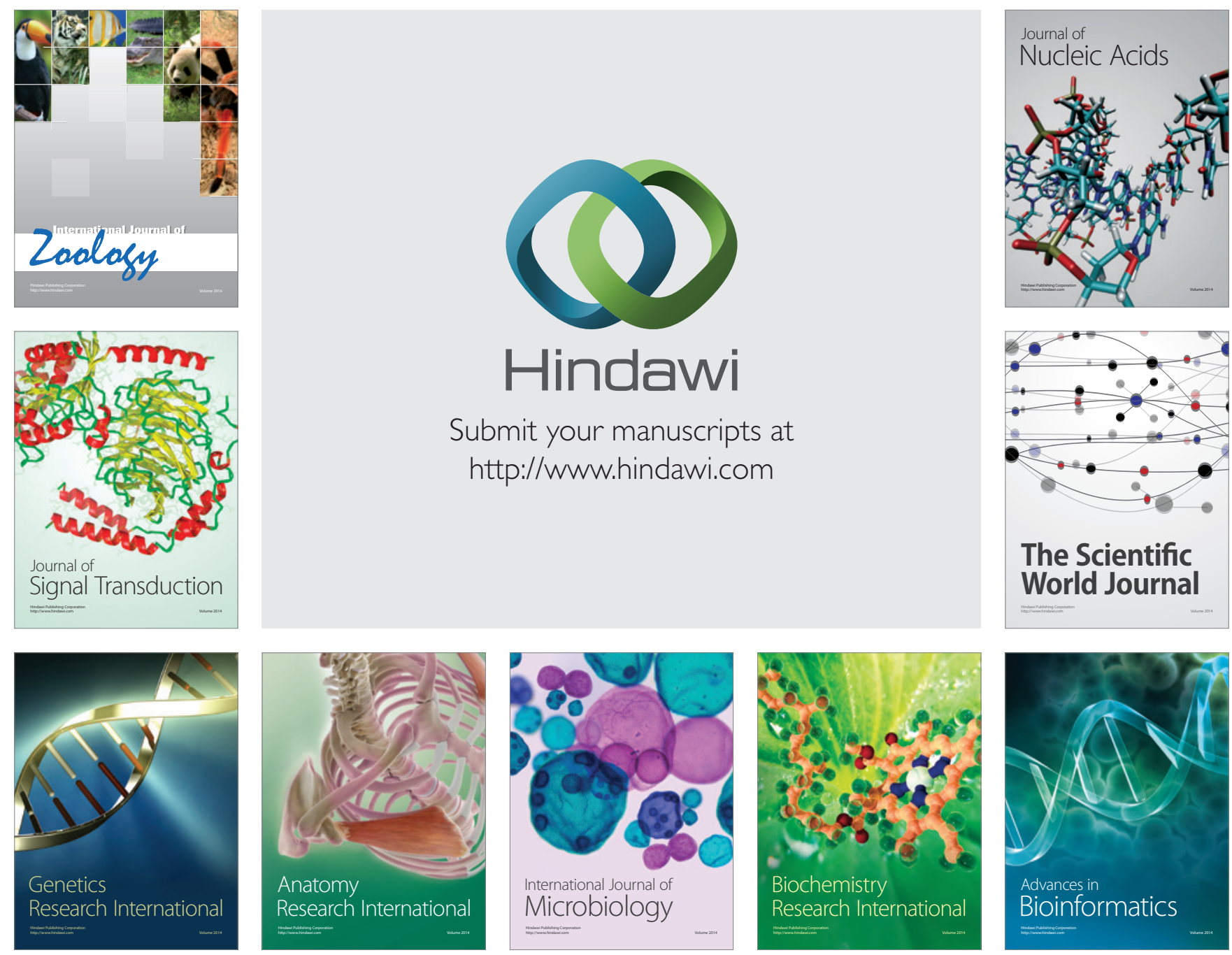

The Scientific World Journal
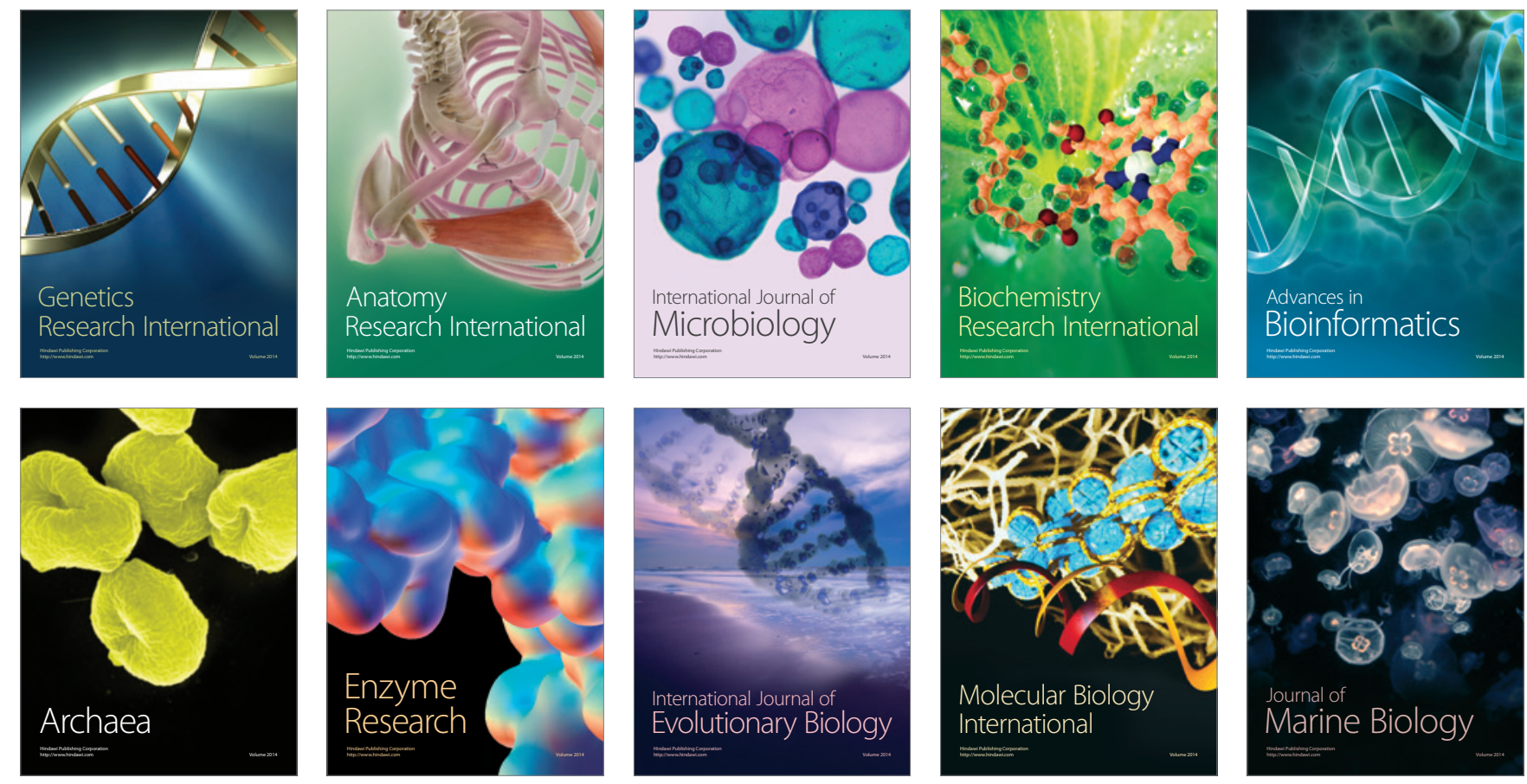